\title{
Rubber friction on smooth surfaces
}

\author{
B.N.J. Persson ${ }^{1}$ and A.I. Volokitin ${ }^{1,2}$ \\ ${ }^{1}$ IFF, FZ-Jülich, 52425 Jülich, Germany and \\ ${ }^{2}$ Samara State Technical University, 443100 Samara, Russia
}

\begin{abstract}
We study the sliding friction for viscoelastic solids, e.g., rubber, on hard flat substrate surfaces. We consider first the fluctuating shear stress inside a viscoelastic solid which result from the thermal motion of the atoms or molecules in the solid. At the nanoscale the thermal fluctuations are very strong and give rise to stress fluctuations in the MPa-range, which is similar to the depinning stresses which typically occur at solid-rubber interfaces, indicating the crucial importance of thermal fluctuations for rubber friction on smooth surfaces. We develop a detailed model which takes into account the influence of thermal fluctuations on the depinning of small contact patches (stress domains) at the rubber-substrate interface. The theory predict that the velocity dependence of the macroscopic shear stress has a bell-shaped form, and that the low-velocity side exhibit the same temperature dependence as the bulk viscoelastic modulus, in qualitative agreement with experimental data. Finally, we discuss the influence of small-amplitude substrate roughness on rubber sliding friction.
\end{abstract}

\section{Introduction}

The friction between rubber and smooth substrate surfaces is a topic of extreme practical importance, e.g., for wiper blades (in particular on hydrophobic glass), rubber O-ring seals, and in the contact region between the tire-rubber and the steel rim on a wheel[1].

When a rubber block is sliding on a very rough substrate, such as a tire on a road surface, the friction is almost entirely due to the energy dissipation in the bulk of the rubber as a result of the fluctuating (in time and space) viscoelastic deformations of the rubber by the substrate asperities [2, 3, 4]. This mechanism becomes unimportant when the substrate is very smooth. In the limiting case of a perfectly smooth substrate, the friction is instead due to local stick-slip events at the sliding interface. Schallamach 5$]$ has proposed a molecular mechanism for the local stick slip, see Fig. 1 where rubber polymer chains at the interface attach to the moving countersurface, stretches, detaches, relaxes, and reattaches to the surface to repeat the cycle (similar models have been studied in Ref. [6, 7]). During each cycle, the elastic energy stored in the polymer chain is dissipated as heat during the (rapid) detachment and relaxation phase, and this is assumed to be the origin of the (macroscopic) friction. However, in our opinion this picture cannot be fully correct. First, for physisorption systems the energy barriers for (vertical) detachment are usually much higher than the energy barriers for lateral sliding 8 , so one would not expect any detachment to occur. Secondly, with respect to the stresses rubber materials are usually exposed to, rubber is nearly incompressible and it is not easy to imagine how single molecules strongly confined at the interface are able to switch between an elongated (stretched) state and a relaxed (curled up) state as indicated in the figure. Furthermore, the sliding friction tend to exhibit the same temperature dependence as the bulk rubber viscoelastic modulus $E(\omega)$ as described by the Williams-Landel-Ferry (WLF) [9] shift factor $a_{T}$. This

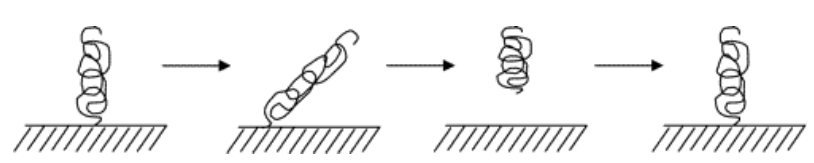

FIG. 1: The classical description of a polymer chain in contact with a lateral moving countersurface. The chain stretches, detaches, relaxes, and reattaches to the surface to repeat the cycle. Adapted from Ref. [20].

indicate the crucial role of the bulk rubber in the friction process.

We believe that the local stick-slip processes, which must occur at the sliding interface, involve relative larger rubber volume elements, always in adhesive contact with the substrate. That is, during sliding small patches (with a diameter of order $D \sim 10-100 \mathrm{~nm}$ ) or stress domains [10] of rubber at the sliding interface perform stick-slip motion: during stick the shear stress at the interface increases continuously with time until the local shear stress reaches a critical depinning stress $\sigma_{\mathrm{c}}$, after which a rapid local slip occur, but with the rubber patch in continuous adhesive contact with the substrate. During the local slip the elastic deformation energy stored in the rubber during the loading phase will be dissipated party inside the rubber (in a volume of order $D^{3}$ ) and partly at the interface. The deformation field in the vicinity of a stress domain of area $\sim D^{2}$ will extend a distance $\sim D$ into the rubber block; we denote this basic unit (volume $\sim D^{3}$ ) as a stress block [see volume elements surrounded by dashed lines in Fig. 2(a)].

Figure 2 illustrate three other mechanisms of rubber friction, which all depend on the rubber-substrate adhesional interaction. Fig. 2(b) illustrate a case where a rubber block is sliding on a smooth wavy substrate. Here we assume only roughness (waviness) on a single length scale, i.e., the substrate bumps have no roughness on length scales smaller than the lateral size of the bumps. In this case, if the adhesional interaction (and the exter- 

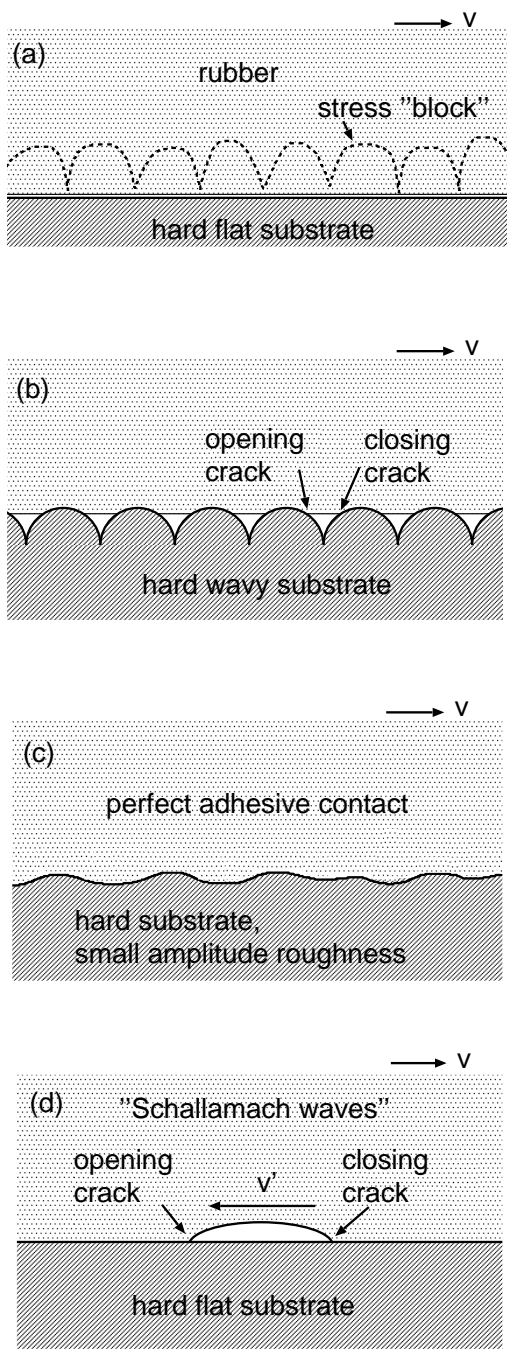

FIG. 2: For different processes where the adhesive rubbersubstrate interaction contribute to rubber friction.

nal applied normal load) is unable to bring the rubber into perfect contact with the substrate, (as in the figure), during sliding opening and closing cracks will occur at the exit and the front of each asperity contact region. In general, negligible bulk viscoelastic energy dissipation occur at the closing crack, while a huge energy dissipation may occur at the opening crack [11, 12]. It has been shown that in some cases the dominant contribution to the friction force arises from the energy "dissipation" at the opening cracks 11, 12.

If the substrate has small-amplitude roughness, the adhesive rubber-substrate interaction may (even in the absence of an external load) lead to complete contact between the rubber and the substrate at the sliding interface [see Fig. 2(c)]. In this case, during sliding viscoelas-

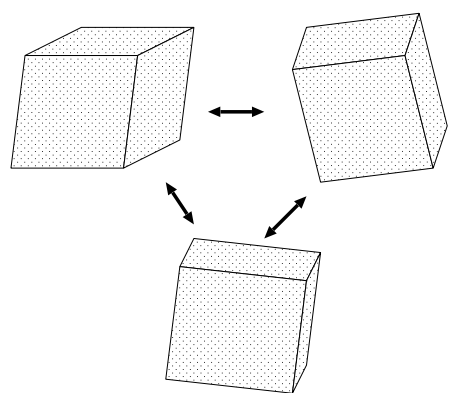

FIG. 3: The thermal motion of the atoms in a solid gives rise to shape fluctuations for a small volume element in the solid (schematic).

tic deformations will occur in the bulk of the rubber in the vicinity of the substrate, which will contribute to the observed friction. This contribution can be calculated using the theory developed in Ref. 2, 13].

Finally, for very soft rubber it has often been observed that detachment waves propagate throughout the entire contact area, from its advancing to the trailing edge, see Fig. 2(d). Schallamach 14] first discovered these waves at "high" sliding velocities and for (elastically) soft rubber. Roberts and Thomas [11, 15] have shown that when such instabilities occur, the frictional stress is mainly due to the energy dissipation at the opening crack, i.e., similar to the situation in Fig. 2(b).

In this paper we study the rubber friction process shown in Fig. 2(a). This is probably the most important rubber friction mechanism in most applications involving very smooth surfaces. In Sec. 2 we consider the fluctuating shear stress inside a viscoelastic solid, which result from the thermal motion of the atoms or molecules in the solid. At the nanoscale the thermal fluctuations are very strong giving stress fluctuations in the MPa-range, which is similar to the depinning stresses which typically occur at solid-rubber interfaces, illustrating the crucial importance of thermal fluctuations for rubber friction on smooth surfaces. In Sec. 3 we develop a detailed model which takes into account the influence of thermal fluctuations on the depinning of small contact areas (stress domains) at the rubber-substrate interface. The theory predict that the velocity dependence of the macroscopic shear stress has a bell-shaped form (see Sec. 4), and that the low-velocity side exhibit the same temperature dependence as the bulk viscoelastic modulus, in qualitative agreement with experimental data (Sec. 5). In Sec. 6 we discuss the role of the surface roughness, which exist even on very smooth surfaces. We show that the countersurfaces used in most rubber sealing applications have such large roughness that the friction observed during sliding at low velocity in many cases may be mainly due to the substrate asperity-induced viscoelastic deformation of the rubber surface. Sec. 7 contains the summary and conclusion. 


\section{Brownian motion in viscoelastic solids}

A small particle in a liquid perform random motion caused by the impact of the surrounding liquid molecules. Similarly, a small volume element in a viscoelastic solid perform shape fluctuations as a result of the thermal motion of the atoms or molecules in the solid, see Fig. 3 Here will will estimate the magnitude of the fluctuating shear stress which act on any (internal) surface in a viscoelastic solid.

Assume that $\mathbf{u}(\mathbf{x}, t)$ is the displacement vector in an infinite viscoelastic solid. The equation of motion for $\mathbf{u}$ is

$$
\rho \frac{\partial^{2} \mathbf{u}}{\partial t^{2}}=\hat{\mu} \nabla^{2} \mathbf{u}+(\hat{\mu}+\hat{\lambda}) \nabla \nabla \cdot \mathbf{u}+\mathbf{f}
$$

where $\hat{\mu}$ and $\hat{\lambda}$ are time integral operators, e.g.,

$$
\hat{\mu} G(t)=\int_{-\infty}^{t} d t^{\prime} \mu\left(t-t^{\prime}\right) G\left(t^{\prime}\right),
$$

and where $\mathbf{f}(\mathbf{x}, t)$ is a randomly fluctuating force density (see below). If we define the Fourier transform

$$
f_{i}(\mathbf{k}, \omega)=\frac{1}{(2 \pi)^{4}} \int d^{3} x d t f_{i}(\mathbf{x}, t) e^{-i(\mathbf{k} \cdot x-\omega t)}
$$

and

$$
f_{i}(\mathbf{x}, t)=\int d^{3} k d \omega f_{i}(\mathbf{k}, \omega) e^{i(\mathbf{k} \cdot x-\omega t)}
$$

we can write

$$
\begin{gathered}
\left\langle f_{i}(\mathbf{k}, \omega) f_{j}\left(\mathbf{k}^{\prime}, \omega^{\prime}\right)\right\rangle=-\frac{k_{\mathrm{B}} T}{\pi \omega}(2 \pi)^{-3} \\
\times\left(\operatorname{Im} \mu(\omega) k^{2} \delta_{i j}+\operatorname{Im} \frac{\mu(\omega)}{1-2 \nu} k_{i} k_{j}\right) \\
\times \delta\left(\mathbf{k}+\mathbf{k}^{\prime}\right) \delta\left(\omega+\omega^{\prime}\right)
\end{gathered}
$$

where $\nu=\lambda / 2(\mu+\lambda)$ and where

$$
\mu(\omega)=\int_{0}^{\infty} d t \mu(t) e^{i \omega t} .
$$

Let us study the fluctuating stress in the solid. The average stress $\left\langle\sigma_{i j}\right\rangle=0$ but the average of the square of any components of $\sigma_{i j}$ will be finite. Here we consider the magnitude of the fluctuating shear stress within some plane which we can take to be the $x y$-plane. Thus we consider

$$
\left\langle\sigma_{\|}^{2}\right\rangle=\left\langle\sigma_{z x}^{2}+\sigma_{z y}^{2}\right\rangle=2\left\langle\sigma_{z x}^{2}\right\rangle
$$

The stress tensor

$$
\sigma_{i j}=\hat{\mu}\left(u_{i, j}+u_{j, i}\right)+\hat{\lambda} u_{k, k} \delta_{i j}
$$

Thus we get

$$
\sigma_{z x}=\hat{\mu}\left(u_{z, x}+u_{x, z}\right)
$$

and

$$
\begin{gathered}
\left\langle\sigma_{\|}^{2}\right\rangle=4\left\langle\left(\hat{\mu} u_{z, x}\right)^{2}+\hat{\mu} u_{z, x} \hat{\mu} u_{x, z}\right\rangle \\
=-4 \int d^{3} k d^{3} k^{\prime} d \omega d \omega^{\prime} \mu(\omega) \mu\left(\omega^{\prime}\right) \\
\quad \times\left(k_{x} k_{x}^{\prime}\left\langle u_{z}(\mathbf{k}, \omega) u_{z}\left(\mathbf{k}^{\prime}, \omega^{\prime}\right)\right\rangle\right. \\
\left.+k_{x} k_{z}^{\prime}\left\langle u_{z}(\mathbf{k}, \omega) u_{x}\left(\mathbf{k}^{\prime}, \omega^{\prime}\right)\right\rangle\right)
\end{gathered}
$$

Using (1) and neglecting inertia effects gives

$$
\begin{gathered}
\mathbf{u}(\mathbf{k}, \omega)=\frac{1}{\mu(\omega) k^{2}+(\mu(\omega)+\lambda(\omega)) \mathbf{k k}} \cdot \mathbf{f}(\mathbf{k}, \omega) \\
=\frac{1}{\mu(\omega) k^{2}}\left(1-\frac{1}{2(1-\nu)} \frac{\mathbf{k k}}{k^{2}}\right) \cdot \mathbf{f}(\mathbf{k}, \omega) .
\end{gathered}
$$

Using (2) and (3) and assuming that $\nu$ is frequency independent gives

$$
\left\langle\sigma_{\|}^{2}\right\rangle=-k_{\mathrm{B}} T C 3(2 \pi)^{-5} \int d^{3} k d \omega \frac{1}{\omega} \operatorname{Im} E(\omega),
$$

where

$$
C=\frac{8 \pi(4-5 \nu)}{45\left(1-\nu^{2}\right)} .
$$

In deriving (4) we have used the relation $\mu=E / 2(1+\nu)$, where $E(\omega)$ is the Young's (viscoelastic) modulus. The shear stress when we only include wavevectors up to $k=$ $2 \pi / D$ (where $D$ is a cut-off length, of order the distance between the cross-links, or larger) is given by

$$
\left\langle\sigma_{\|}^{2}\right\rangle=-k_{\mathrm{B}} T C D^{-3} \frac{2}{\pi} \int_{0}^{\infty} d \omega \frac{1}{\omega} \operatorname{Im} E(\omega)
$$

Using the sum rule 11,12

$$
\frac{2}{\pi} \int_{0}^{\infty} d \omega \frac{1}{\omega} \operatorname{Im} E(\omega)=E_{0}-E_{\infty}
$$

where $E_{0}=E(0)$ and $E_{\infty}=E(\infty)$ are the low- and high-frequency rubber modulus (both real), we get

$$
\bar{\sigma}_{\|}^{2} \equiv\left\langle\sigma_{\|}^{2}\right\rangle=k_{\mathrm{B}} T C D^{-3}\left(E_{\infty}-E_{0}\right)
$$

Assuming $\nu=0.5$ gives $2 \pi C / 3 \approx 1.1$. In a typical case $E_{\infty} \approx 10^{9} \mathrm{~Pa}>E_{0}$ and if $D=10 \mathrm{~nm}$ we get at room temperature $\bar{\sigma}_{\|} \approx 1 \mathrm{MPa}$, which (typically) is of the same order of magnitude as the depinning stress at a rubber-substrate interface. 
It is also interesting to estimate the fluctuation in the displacement $\mathbf{u}_{\|}$and the fluctuation in the strain. Using the same approach as above one obtain

$$
\left\langle u_{\|}^{2}\right\rangle=\left\langle u_{x}^{2}+u_{y}^{2}\right\rangle=k_{\mathrm{B}} T C^{\prime} D^{-1} \frac{2}{\pi} \int_{0}^{\infty} d \omega \frac{1}{\omega} \operatorname{Im}\left(\frac{1}{E(\omega)}\right)
$$

where

$$
C^{\prime}=\frac{2(5-6 \nu)(1+\nu)}{3(1-\nu)}
$$

If we use the sum rule 11,12

$$
\frac{2}{\pi} \int_{0}^{\infty} d \omega \frac{1}{\omega} \operatorname{Im}\left(\frac{1}{E(\omega)}\right)=\frac{1}{E_{0}}-\frac{1}{E_{\infty}}
$$

we get

$$
\left\langle u_{\|}^{2}\right\rangle=k_{\mathrm{B}} T C^{\prime} D^{-1}\left(\frac{1}{E_{0}}-\frac{1}{E_{\infty}}\right)
$$

In a similar way one can calculate the average of the square of the strain

$$
\left\langle\epsilon_{\|}^{2}\right\rangle=\left\langle\epsilon_{z x}^{2}+\epsilon_{z y}^{2}\right\rangle=k_{\mathrm{B}} T C^{\prime \prime} D^{-3}\left(\frac{1}{E_{0}}-\frac{1}{E_{\infty}}\right)
$$

where

$$
C^{\prime \prime}=\frac{32 \pi(4-5 \nu)(1+\nu)^{2}}{45\left(1-\nu^{2}\right)}
$$

With $E_{0}=1 \mathrm{MPa}$ and $D=10 \mathrm{~nm}$ one get at room temperature $\bar{u}_{\|} \approx 1 \mathrm{~nm}$ and $\bar{\epsilon}_{\|} \approx 0.3$.

The analysis above indicate that, on the length scale of $\sim 10 \mathrm{~nm}$, very large strain and stress fluctuations occur in normal rubber. The stress fluctuations are of similar magnitude as the (typical) rubber-substrate depinning stress, suggesting that thermally excited transitions over the (lateral) energy barriers play a crucial role in rubber friction on smooth substrates. However, the stress fluctuations $(\sim 1 \mathrm{MPa})$ are negligible compared to the stress necessary for detaching a rubber patch in the normal direction, since the latter is determined by the adhesional stress 16, 17] which typically is of order $1 \mathrm{GPa}$. Thus, rubber sliding on smooth surfaces never involves (thermally activated) detachment of rubber from the substrate surface, but only (lateral) sliding of rubber patches (the lateral depinning stress is typically $100-1000$ times smaller than the adhesional stress). [Detached areas may form and propagate at the interface (as for Schallamach waves, see fig. 2(d)), but in these cases they are generated by the external applied stress, and depend on the shape of the bodies; the detached regions usually form at the edge of the rubber-substrate contact region, where elastic instabilities (e.g., "buckling") of the rubber may occur [18.] In the next section we will develop a model of rubber sliding friction based on the picture presented above.

\section{Theory}

We consider a rubber block with a smooth flat surface sliding on a perfectly smooth and flat substrate. We assume that the adhesive rubber-substrate interaction will result in perfect contact between the two solids (i.e., we assume that no Schallamach waves occur at the interface). During sliding at low velocities, small (nanometer sized) regions (stress blocks) at the sliding interface will perform stick-slip motion. We model the real physical system in Fig. 4(a) with the block-spring model shown in Fig. 4(b). However, the springs in the model are not elastic springs but viscoelastic springs determined by the viscoelastic modulus of the rubber (see below). Furthermore, the blocks in the model experience not only the stress from the substrate and the forces from the springs, but also randomly fluctuating (in time) forces derived from the thermal motion of the molecules in the solid. The strength of the fluctuating forces is determined by the temperature and by the viscoelastic properties of the springs via the fluctuation-dissipation theorem. The combination of the (thermal) fluctuating forces, and the forces derived from the external pulling of the upper surface of the rubber block, and the stress acting on the bottom surface of the block from the substrate, determine the motion of the stress blocks.

\subsection{Basic equations}

We assume that the motion at low sliding velocity occur by a thermally activated process, where small surface areas or "patches" of rubber at the interface perform stick-slip motion. We will refer to these "patches" as stress domains. The stress domains are pinned by the substrate potential, and we assume that some characteristic shear stress $\sigma_{c}$ must be reached before local slip can occur. Thus, the pinning force $F_{c}=\sigma_{c} D^{2}$, where $D$ is the characteristic linear size of a stress domain.

The equation of motion for the coordinate $q_{i}$ of the stress domain $i$ is assumed to be

$$
\begin{aligned}
m \ddot{q}_{i} & =\hat{k}_{z}\left(x-q_{i}\right)+\hat{k}_{x}\left(q_{i+1}-q_{i}\right) \\
& +\hat{k}_{x}\left(q_{i-1}-q_{i}\right)+f_{i}+F_{i}
\end{aligned}
$$

where $x=v t$ and where $\hat{k}_{z}$ is a time integral operator

$$
\hat{k}_{z} x(t)=\int_{-\infty}^{t} d t^{\prime} k_{z}\left(t-t^{\prime}\right) x\left(t^{\prime}\right)
$$

and similar for $\hat{k}_{x}$. We consider $N$ stress domains, $i=1, \ldots, N$, and assume periodic boundary conditions so that $q_{0}=q_{N}$. In $(5), f_{i}(t)$ is a stochastically fluctuating force which result from the thermal motion of the rubber molecules. The force $F_{i}$ act on the stress domain $i$ from the substrate and is defined as follows: When the stress domain $i$ slips, then $F_{i}=-m \eta \dot{q}_{i}$. In the pinned 


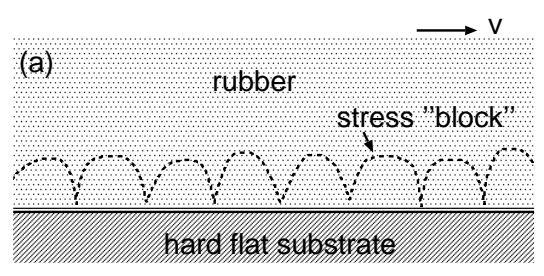

(b) model viscoelastic spring

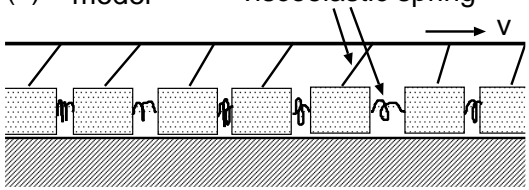

FIG. 4: (a) Rubber block in adhesive contact with a flat substrate. During sliding at low velocities, small rubber volume elements (stress blocks) perform stick-slip motion. (b) The model used in the mathematical description of the system in (a). The viscoelastic springs $k$ are determined by the rubber viscoelastic modulus and by the lateral size $D$ of the stress blocks via $k(\omega) \approx E(\omega) D$.

state, $F_{i}$ is just large enough to balance the total force exerted by the stress block on the substrate:

$$
F_{i}=-\hat{k}_{z}\left(x-q_{i}\right)-\hat{k}_{x}\left(q_{i+1}-q_{i}\right)-\hat{k}_{x}\left(q_{i-1}-q_{i}\right)-f_{i}
$$

Slip will start when $\left|F_{i}\right|$ reaches a critical value $F_{c}$, either as a result of the external applied force or as a result of a large enough thermal fluctuation, or, in general, as a combination of both these effects.

We define the Fourier transform

$$
\begin{gathered}
x(\omega)=\frac{1}{2 \pi} \int d t x(t) e^{i \omega t} \\
x(t)=\int d \omega x(\omega) e^{-i \omega t}
\end{gathered}
$$

The fluctuating force $f_{i}(t)$ result from the thermal motion of the rubber molecules and must satisfy the fluctuationdissipation theorem. That is, if we write

$$
\hat{K}_{i j} q_{j}=\hat{k}_{z} q_{i}-\hat{k}_{x}\left(q_{i+1}-q_{i}\right)-\hat{k}_{x}\left(q_{i-1}-q_{i}\right)
$$

then from the theory of Brownian motion (see Appendix A)

$$
\left\langle f_{i}(\omega) f_{j}\left(\omega^{\prime}\right)\right\rangle=-\frac{k_{B} T}{\pi \omega} \operatorname{Im} K_{i j}(\omega) \delta\left(\omega+\omega^{\prime}\right)
$$

where

$$
K_{i j}(\omega)=\int_{0}^{\infty} d t K_{i j}(t) e^{i \omega t}
$$

and (for $t>0$ ):

$$
K_{i j}(t)=\frac{1}{2 \pi} \int d \omega K_{i j}(\omega) e^{-i \omega t} .
$$

If we write the elastic modulus as 28]

$$
E(\omega)=E_{\infty}\left(1-\sum_{n} \frac{h_{n}}{1-i \omega \tau_{n}}\right)
$$

we get

$$
K_{i j}(\omega)=K_{i j}^{\infty}-K_{i j}^{\infty} \sum_{n} \frac{h_{n}}{1-i \omega \tau_{n}},
$$

where

$$
K_{i j}^{\infty}=\left(k_{z}^{\infty}+2 k_{x}^{\infty}\right) \delta_{i j}-k_{x}^{\infty}\left(\delta_{i, j+1}+\delta_{i, j-1}\right) .
$$

Eq. (12) gives

$$
\operatorname{Im} K_{i j}(\omega)=-K_{i j}^{\infty} \sum_{n} \frac{h_{n} \tau_{n} \omega}{\left|1-i \omega \tau_{n}\right|^{2}}
$$

Substituting (13) in (11) gives

$$
\left\langle f_{i}(\omega) f_{j}\left(\omega^{\prime}\right)\right\rangle=\frac{k_{B} T}{\pi} K_{i j}^{\infty} \sum_{n} \frac{h_{n} \tau_{n}}{\left|1-i \omega \tau_{n}\right|^{2}} \delta\left(\omega+\omega^{\prime}\right)
$$

We can write

$$
\begin{gathered}
-K_{i j}(\omega) q_{j}(\omega)+k_{z}(\omega) x(\omega) \\
=-K_{i j}^{\infty} q_{j}(\omega)+k_{z}^{\infty} x(\omega)-\sum_{n} h_{n} u_{n i}(\omega)
\end{gathered}
$$

where

$$
u_{n i}(\omega)=\frac{-1}{1-i \omega \tau_{n}} K_{i j}^{\infty} q_{j}(\omega)+\frac{1}{1-i \omega \tau_{n}} k_{z}^{\infty} x(\omega)
$$

or

$$
u_{n i}(t)+\tau_{n} \dot{u}_{n i}(t)=-K_{i j}^{\infty} q_{j}(t)+k_{z}^{\infty} x+g_{n i}(t)
$$

Here we have added a stochastically fluctuating force on the right hand side of (17) which we can choose so as to reproduce the fluctuating force $f_{i}(t)$ in Eq. (5). That is, if we choose $g_{n i}(t)$ appropriately, we can remove the force $f_{i}(t)$ in Eq. (5). To this end we must choose

$$
f_{i}(\omega)=-\sum_{n} \frac{h_{n} g_{n j}(\omega)}{1-i \omega \tau_{n}}
$$

with

$$
g_{n j}(\omega)=N^{-1 / 2} \operatorname{Re} \sum_{k} M_{k n} e^{i k x_{j}} \xi_{k n}
$$


where

$$
M_{k n}=\left(\frac{k_{B} T \tau_{n} K_{k}^{\infty}}{\pi h_{n}}\right)^{1 / 2}
$$

with the $k$-sum over

$$
k=\frac{2 \pi}{D} \frac{r}{N}, \quad r=0,1,2, \ldots, N-1
$$

Note that

$$
K_{k}^{\infty}=k_{z}^{\infty}+k_{x}^{\infty} 2[1-\cos (k D)]
$$

is the (discrete) Fourier transform of $K_{i j}^{\infty}$. In (19), $\xi_{k n}$ are complex Gaussian random variables:

$$
\xi_{k n}=\zeta_{k n}+i \eta_{k n}
$$

where

$$
\begin{gathered}
\left\langle\zeta_{k n}(\omega) \zeta_{k^{\prime} n^{\prime}}\left(\omega^{\prime}\right)\right\rangle=\delta_{n n^{\prime}} \delta_{k k^{\prime}} \delta\left(\omega+\omega^{\prime}\right) \\
\left\langle\eta_{k n}(\omega) \eta_{k^{\prime} n^{\prime}}\left(\omega^{\prime}\right)\right\rangle=\delta_{n n^{\prime}} \delta_{k k^{\prime}} \delta\left(\omega+\omega^{\prime}\right) \\
\left\langle\zeta_{k n}(\omega) \eta_{k^{\prime} n^{\prime}}\left(\omega^{\prime}\right)\right\rangle=0
\end{gathered}
$$

Using (19) and (22) gives

$$
g_{n j}(\omega)=N^{-1 / 2} \sum_{k} M_{k n}\left[\zeta_{k n} \cos \left(k x_{j}\right)-\eta_{k n} \sin \left(k x_{j}\right)\right]
$$

Using (23)-(25) this gives

$$
\begin{gathered}
\left\langle g_{n l}(\omega) g_{n^{\prime} l^{\prime}}\left(\omega^{\prime}\right)\right\rangle \\
=N^{-1} \sum_{k} M_{k n}^{2} \cos \left[k\left(x_{l}-x_{l^{\prime}}\right)\right] \delta_{n n^{\prime}} \delta\left(\omega+\omega^{\prime}\right) \\
=N^{-1} \sum_{k} M_{k n}^{2} e^{i k\left(x_{l}-x_{l^{\prime}}\right)} \delta_{n n^{\prime}} \delta\left(\omega+\omega^{\prime}\right) \\
=\frac{k_{B} T \tau_{n}}{\pi h_{n} N} \sum_{k} K_{k}^{\infty} e^{i k\left(x_{l}-x_{l^{\prime}}\right)} \delta_{n n^{\prime}} \delta\left(\omega+\omega^{\prime}\right) \\
=\frac{k_{B} T \tau_{n}}{\pi h_{n}} K_{l l^{\prime}}^{\infty} \delta_{n n^{\prime}} \delta\left(\omega+\omega^{\prime}\right)
\end{gathered}
$$

Using (18) and (27) gives

$$
\left\langle f_{i}(\omega) f_{j}\left(\omega^{\prime}\right)\right\rangle=\frac{k_{B} T}{\pi} K_{i j}^{\infty} \sum_{n} \frac{h_{n} \tau_{n}}{\left|1-i \omega \tau_{n}\right|^{2}} \delta\left(\omega+\omega^{\prime}\right)
$$

which agree with (14).

Let us summarize the basic equations:

$$
m \ddot{q}_{i}=k_{z}^{\infty}\left(x-q_{i}\right)+k_{x}^{\infty}\left(q_{i+1}+q_{i-1}-2 q_{i}\right)
$$

$$
-\sum_{n} h_{n} u_{n i}+F_{i}
$$

where

$$
\begin{gathered}
u_{n i}(t)+\tau_{n} \dot{u}_{n i}(t)=k_{z}^{\infty}\left(x-q_{i}\right) \\
+k_{x}^{\infty}\left(q_{i+1}+q_{i-1}-2 q_{i}\right)+g_{n i}(t)
\end{gathered}
$$

The random force

$$
g_{n j}(\omega)=N^{-1 / 2} \operatorname{Re} \sum_{k} M_{k n} e^{i k x_{j}} \xi_{k n}(\omega)
$$

where

$$
M_{k n}=\left(\frac{k_{B} T \tau_{n} K_{k}^{\infty}}{\pi h_{n}}\right)^{1 / 2} .
$$

\subsection{Numerical implementation}

If $D$ is the lateral size of a stress block, then the mass of a stress block $m=\rho D^{3}$. We introduce the spring constants $k_{z}^{\infty}=\alpha_{z} k^{*}$ and $k_{x}^{\infty}=\alpha_{x} k^{*}$ where $k^{*}=D E_{\infty}$, and where $\alpha_{x}$ and $\alpha_{z}$ are of order unity. We measure time in units of $\tau=\left(m / k^{*}\right)^{1 / 2}$ and distance in unit of $l=F_{c} / k^{*}$ where $F_{c}=\sigma_{c} D^{2}$ is the stress block pinning force. We also measure $u$ in units of $F_{c}$ and $x$ in units of $l$. In these units we get

$$
\begin{gathered}
\ddot{q}_{i}=\alpha_{z}^{\infty}\left(x-q_{i}\right)+\alpha_{x}^{\infty}\left(q_{i+1}+q_{i-1}-2 q_{i}\right) \\
-\sum_{n} h_{n} u_{n i}+F_{i} / F_{c}
\end{gathered}
$$

where

$$
\begin{gathered}
u_{n i}(t)+\left(\tau_{n} / \tau\right) \dot{u}_{n i}(t)=\alpha_{z}\left(x-q_{i}\right) \\
+\alpha_{x}\left(q_{i+1}+q_{i-1}-2 q_{i}\right)+g_{n i}(t)
\end{gathered}
$$

The random force

$$
g_{n j}(\omega)=N^{-1 / 2} \operatorname{Re} \sum_{k} M_{k n}^{*} e^{i k x_{j}} \xi_{k n}(\omega)
$$

where

$$
M_{k n}^{*}=M_{k n} / F_{c}=\left(\frac{k_{B} T \tau_{n} K_{k}^{\infty}}{2 \pi \Delta E k^{*} h_{n}}\right)^{1 / 2},
$$

where $\Delta E=k^{*} l^{2} / 2$. Note also that

$$
\left\langle\zeta_{k n}(\omega) \zeta_{k^{\prime} n^{\prime}}\left(\omega^{\prime}\right)\right\rangle=\delta_{n n^{\prime}} \delta_{k k^{\prime}} \delta\left(\omega+\omega^{\prime}\right)
$$

gives

$$
\left\langle\zeta_{k n}(t) \zeta_{k^{\prime} n^{\prime}}\left(t^{\prime}\right)\right\rangle=2 \pi \delta_{n n^{\prime}} \delta_{k k^{\prime}} \delta\left(t-t^{\prime}\right)
$$


In order to numerically integrate the equations (33) and (34) we discretize time with the step length $\delta t$. The (fluctuating) force $\delta g_{n i}$ to be used for each time-step in (34) can be written as

$$
\delta g_{n j}=N^{-1 / 2} \operatorname{Re} \sum_{k} M_{k n}^{*} e^{i k x_{j}} \int_{t}^{t+\delta t} d t^{\prime} \xi_{k n}\left(t^{\prime}\right)
$$

But if

$$
\left\langle\zeta(t) \zeta\left(t^{\prime}\right)\right\rangle=2 \pi \delta\left(t-t^{\prime}\right)
$$

we get

$$
\left\langle(\delta \zeta)^{2}\right\rangle=\int_{t}^{t+\delta t} d t^{\prime} d t^{\prime \prime}\left\langle\zeta\left(t^{\prime}\right) \zeta\left(t^{\prime \prime}\right)\right\rangle=2 \pi \delta t
$$

Thus we can write $\delta \zeta=(2 \pi \delta t)^{1 / 2} G$ where $G$ is a Gaussian random number with $\left\langle G^{2}\right\rangle=1$. Thus, we take

$$
\delta g_{n j}=N^{-1 / 2} \operatorname{Re} \sum_{k} M_{k n}^{*} e^{i k x_{j}}(2 \pi \delta t)^{1 / 2}\left(G_{k n}^{(1)}+i G_{k n}^{(2)}\right)
$$

where $G_{k n}^{(1)}$ and $G_{k n}^{(2)}$ are Gaussian random numbers. We can also write

$$
\delta g_{n j}=N^{-1 / 2} \operatorname{Re} \sum_{k} \bar{M}_{k n} e^{i k x_{j}}\left(G_{k n}^{(1)}+i G_{k n}^{(2)}\right)
$$

where

$$
\bar{M}_{k n}=\left(\frac{k_{B} T}{\Delta E} \frac{\tau_{n} \delta t}{\tau^{2}} \frac{K_{k}^{\infty}}{h_{n} k^{*}}\right)^{1 / 2} .
$$

In the calculations presented below we have assumed that a sliding block return to the pinned state when the shear stress $|\sigma|<\sigma_{c 1}=\lambda \sigma_{c}$, where $\sigma_{c}=F_{c} / D^{2}$ is the depinning stress and $\lambda<1$. We assume that when the shear stress has decreased below $\sigma_{c 1}$ the block return to the pinned state with the probability rate $w$, and we use random number to determine when the transition actually takes place. Thus, if $r$ is a random number uniformly distributed in the interval $[0,1]$, then if $|\sigma|<\sigma_{c 1}$ we assume that the stress block return to the pinned state during the time interval $\delta t$ (the time integration step length) if $w \delta t>r$. In the simulations below we use $w \leq 10^{12} \mathrm{~s}^{-1}$ and $\lambda=0.1$. We use $\delta \approx 10^{-13} \mathrm{~s}$ in most of our simulations so that the condition $w \delta t<<1$ is satisfied, and the results presented below does not depend on the time step $\delta t$. The calculation of $\delta g_{n j}$ (Eq. (35)) is conveniently performed using the Fast Fourier Transform method.

\section{Numerical results}

We now present numerical results for styrenebutadiene rubber both with and without filler.

Fig. 5] a) shows the frictional shear stress $\sigma_{\mathrm{f}}$ and (b) the fraction of slipping surface area $N / N_{0}$ as a function of the logarithm (with 10 as basis) of the sliding velocity
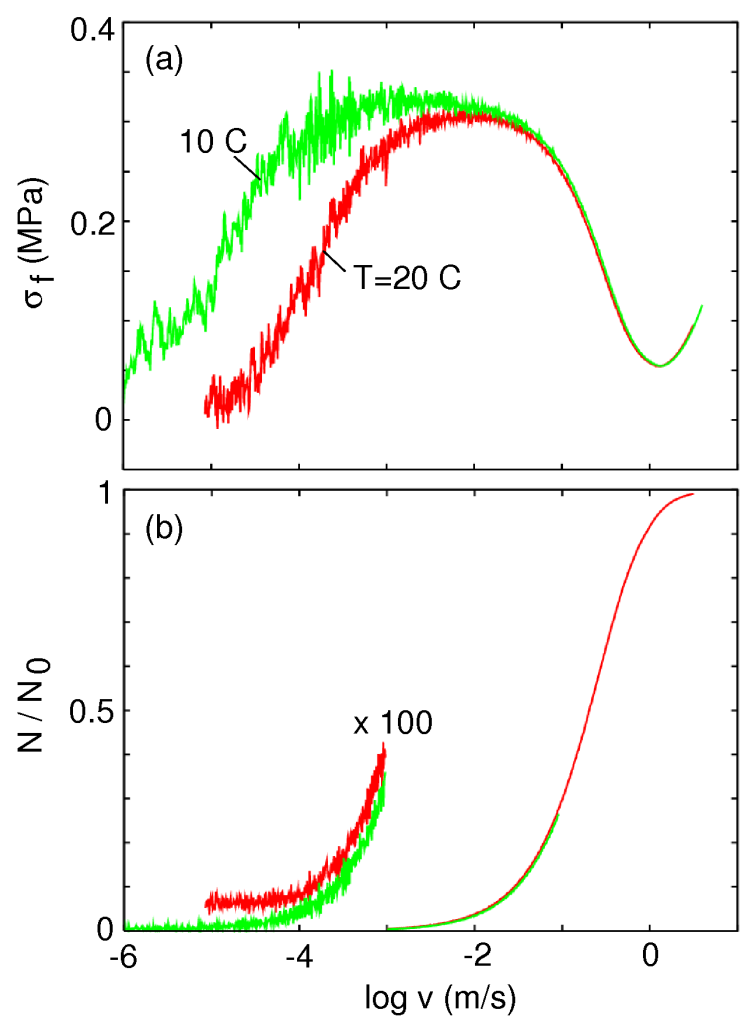

FIG. 5: (a) The frictional shear stress $\sigma_{\mathrm{f}}$ and (b) the fraction of slipping surface area $N / N_{0}$ as a function of the logarithm (with 10 as basis) of the sliding velocity of the rubber block. For Styrene butadiene copolymer with $60 \%$ carbon black and for two different temperatures, $T=10{ }^{\circ} \mathrm{C}$ and $20^{\circ} \mathrm{C}$. In the calculation we have used the (zero-temperature) depinning stress $\sigma_{\mathrm{c}}=1 \mathrm{MPa}$ and the stress block size $D=30 \mathrm{~nm}$. The number of stress blocks was 128 . The viscous friction coefficient during steady sliding $\eta=0.03$ (natural units) and the critical stress below which steady sliding becomes metastable $\sigma_{\mathrm{c} 1}=0.1 \sigma_{\mathrm{c}}=0.1 \mathrm{MPa}$. The probability rate per unit time to return to the pinned state when $\sigma<\sigma_{\mathrm{c} 1}$ is $w=2 \times 10^{10} \mathrm{~s}^{-1}$.

of the rubber block. The results are for styrene butadiene copolymer with $60 \%$ carbon black, and for two different temperatures, $T=10^{\circ} \mathrm{C}$ and $20^{\circ} \mathrm{C}$. Note that the low velocity part $\left(v<v_{1}\right.$, where $v_{1} \approx 10^{-2} \mathrm{~m} / \mathrm{s}$ is the velocity at which the friction is maximal) of the friction curve is shifted by $\sim 1$ decade towards lower velocities when the temperature is reduced from 20 to $10^{\circ} \mathrm{C}$. This is identical to the change in the (bulk) viscoelastic shift factor $a_{T}$, which change by a factor of $\sim 10$ during the same temperature change. Fig. [5(b) shows that the number of moving stress blocks is basically temperature independent for sliding velocities $v>v_{1} \approx 10^{-2} \mathrm{~m} / \mathrm{s}$, i.e., for $v>v_{1}$ the fluctuating force arising from the finite temperature has a negligible influence on the friction force. For $v<v_{1}$ more stress blocks are depinned at the higher temperature because the fluctuating force is larger at the higher temperature. Note also that the maximal shear stress $\sigma_{\mathrm{f}} \approx 0.3 \mathrm{MPa}$ is about $30 \%$ of the depinning stress 

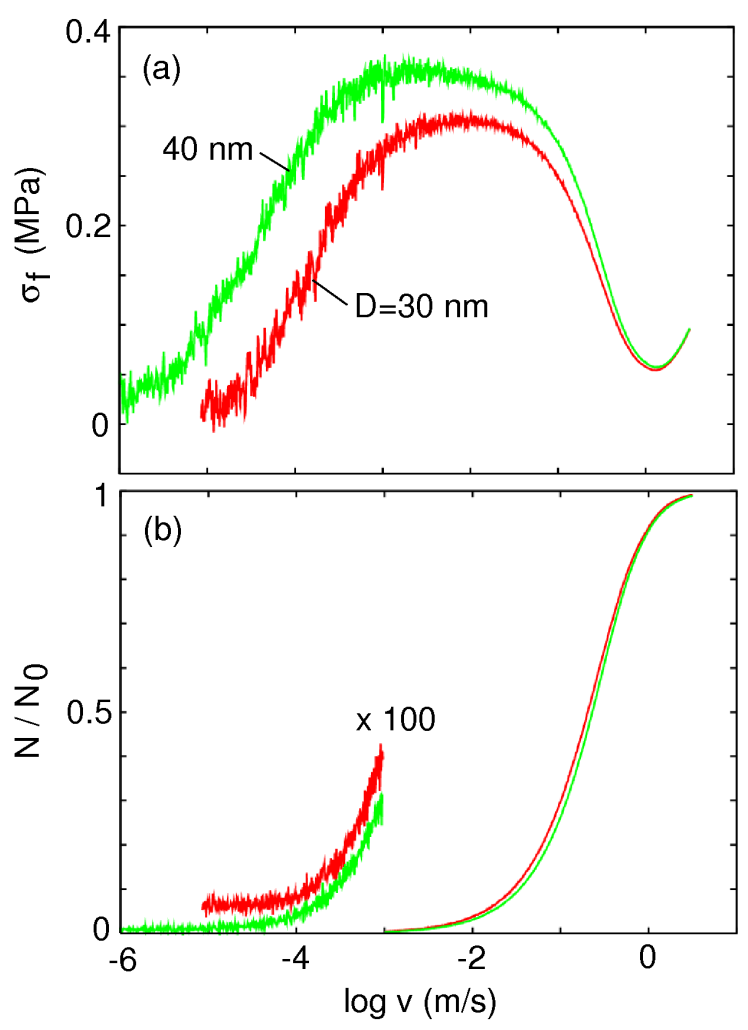

FIG. 6: The frictional shear stress $\sigma_{\mathrm{f}}$ as a function of the logarithm of the sliding velocity of the rubber block. For the temperature $T=20{ }^{\circ} \mathrm{C}$ and for the stress block sizes $D=30$ and $40 \mathrm{~nm}$. All other parameters as in Fig. 5.

$\sigma_{c}=1 \mathrm{MPa}$.

Fig. [a) shows the frictional shear stress $\sigma_{\mathrm{f}}$ and (b) the fraction of sliding blocks, as a function of the logarithm of the sliding velocity. In the calculation we have used $T=20^{\circ} \mathrm{C}$ and the stress block sizes $D=30$ and $40 \mathrm{~nm}$. When the stress block size increases the pinning force $\sigma_{c} D^{2}$ increases, and it is necessary to go to lower sliding velocities in order for temperature effects to manifest them-self (as a decrease in the frictional shear stress).

The linear size of the stress blocks, $D$, is most likely determined by the elastic modulus and the depinning shear stress $\sigma_{c}$ as follows. The stress block is the smallest unit which is able to slide as a coherent unit and can be determined as follows. The depinning force is $F_{c}=\sigma_{c} D^{2}$. If the stress $\sigma$ act at the bottom surface of a stress block it will move a distance $x$ determined by $k x=\sigma D^{2}$ where $k \approx E D$, where $E$ is the elastic modulus. The stress block experience a quasi-periodic pinning potential from the substrate, characterized by a lattice constant $a$ of order a few Angstroms. Thus, the stress block will in general be able to occupy a "good" binding position in the corrugated substrate potential only if $k a$ is less than the pinning potential [19]. The condition $k a=F_{c}$ gives the size $D$ of the pinned domains. Using $F_{c}=\sigma_{c} D^{2}$ and

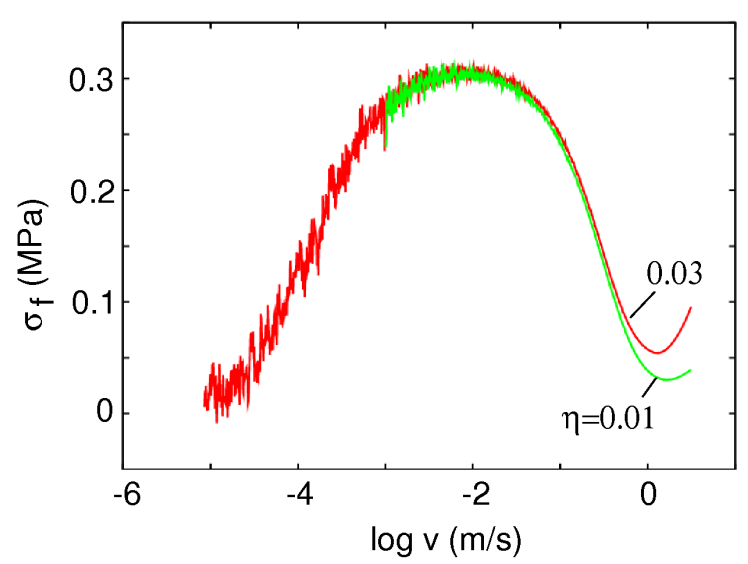

FIG. 7: The frictional shear stress $\sigma_{\mathrm{f}}$ as a function of the logarithm of the sliding velocity of the rubber block. For the temperature $T=20{ }^{\circ} \mathrm{C}$ and for the viscous friction coefficients $\eta=0.03$ and 0.01 (natural units). All other parameters as in Fig. 5.

$k \approx E D$ this gives

$$
D \approx E a / \sigma_{c}
$$

In a typical case $E \approx 10 \mathrm{MPa}, \sigma_{c} \approx 1 \mathrm{MPa}$ and $a$ a few Angstroms, giving $D=30 \mathrm{~nm}$. Since $D$ increases when the elastic modulus increases, the present theory indicate that the friction should increase with increasing elastic modulus. This is exactly what has been observed in friction studies for smooth surfaces (see below). Since the elastic modulus $E$ depends on frequency, during sliding the size $D$ of the stress domains may depend on the sliding velocity, but we have not taken this effect into account in this paper.

Fig. 7 shows the frictional shear stress $\sigma_{\mathrm{f}}$ as a function of the logarithm of the sliding velocity. Results are shown for two different viscous friction coefficients $\eta=0.03$ and 0.01 (natural units). As expected, $\eta$ is only important at relative high sliding velocities.

Fig. 8 shows how $\sigma_{\mathrm{f}}$ depend on the sliding velocity for two different values $(0.1$ and $0.2 \mathrm{MPa})$ of the critical stress $\sigma_{\mathrm{c} 1}$ below which the sliding patch can return to the pinned state.

Fig. 9] shows the frictional shear stress $\sigma_{\mathrm{f}}$ as a function of the logarithm of the sliding velocity of the rubber block for two different values $\left(2 \times 10^{10}\right.$ and $\left.10^{12} \mathrm{~s}^{-1}\right)$ of the probability rate per unit time to return to the pinned state when $\sigma<\sigma_{\mathrm{c} 1}$. Fig. 10] shows similar results for two different values (1 and 0.7 MPa) of the depinning stress $\sigma_{\mathrm{c}}$.

Fig [1] shows the frictional shear stress $\sigma_{\mathrm{f}}$ as a function of the logarithm of the sliding velocity of the rubber block for an unfilled styrene butadiene (SB) copolymer for two different values (30 and $40 \mathrm{~nm}$ ) of the size $D$ of a pinned region. 


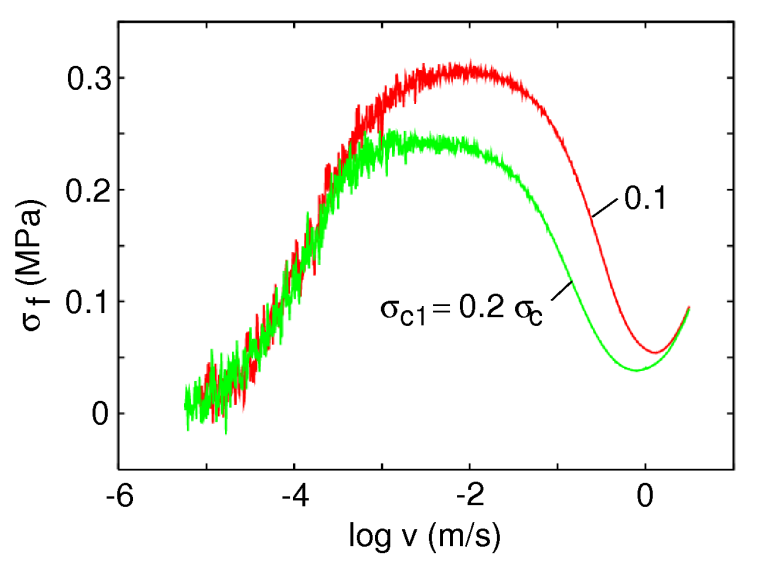

FIG. 8: The frictional shear stress $\sigma_{\mathrm{f}}$ as a function of the logarithm of the sliding velocity of the rubber block. For the temperature $T=20^{\circ} \mathrm{C}$ and for the critical stresses (below which steady sliding becomes metastable) $\sigma_{\mathrm{c} 1}=0.1 \sigma_{\mathrm{c}}=0.1 \mathrm{MPa}$ and $0.2 \mathrm{MPa}$. All other parameters as in Fig. 5 .

\section{Discussion}

Vorvolakos and Chaudhury 20] have performed a very detailed experimental study of sliding friction for silicon rubber on hard flat (passivated) substrates (see also Ref. 21, 22, 23] for other studies of elastomer friction). They used silicon rubbers with many different (low-frequency) elastic modulus $E$.

In Fig. 12 we show the velocity dependence of the shear stress as measured at different temperatures $T=$ 298 (open circles), 318 (gray circles) and $348 \mathrm{~K}$ (black circles) for a silicon rubber with the low-frequency elastic modulus $E \approx 5 \mathrm{MPa}$. The experimental data on the low-velocity side can be shifted to a single curve when plotted as a function of $v a_{T}$, see Ref. 20]. This is in accordance with our model calculations (see Sec. 4) and shows the direct involvement of the rubber bulk in the sliding dynamics.

Vorvolakos and Chaudhury 20 have shown that the frictional shear stress for all the studied rubbers increases with increasing elastic modulus. This is in qualitative agreement with our theory since, as explained in Sec. 4, as $E$ increases we expect the linear size $D$ of the stress domains to increase, which will increase the sliding friction (see Fig. 6] and 11).

In the experiments by Grosch 21 it was observed that the friction on smooth surfaces has a bell-like shape with a maximum at some characteristic velocity $v=v_{1}$. We observe the same general behavior, but with some important differences. Thus, the experimental data of Grosch was observed to obey the WLF transform. That is, the full friction curve could be constructed by performing measurements in a very limited velocity range and at different temperatures, and then use the WLF transform to shift the measured data to a single temperature. We also find that our calculated friction obey the WLF transform

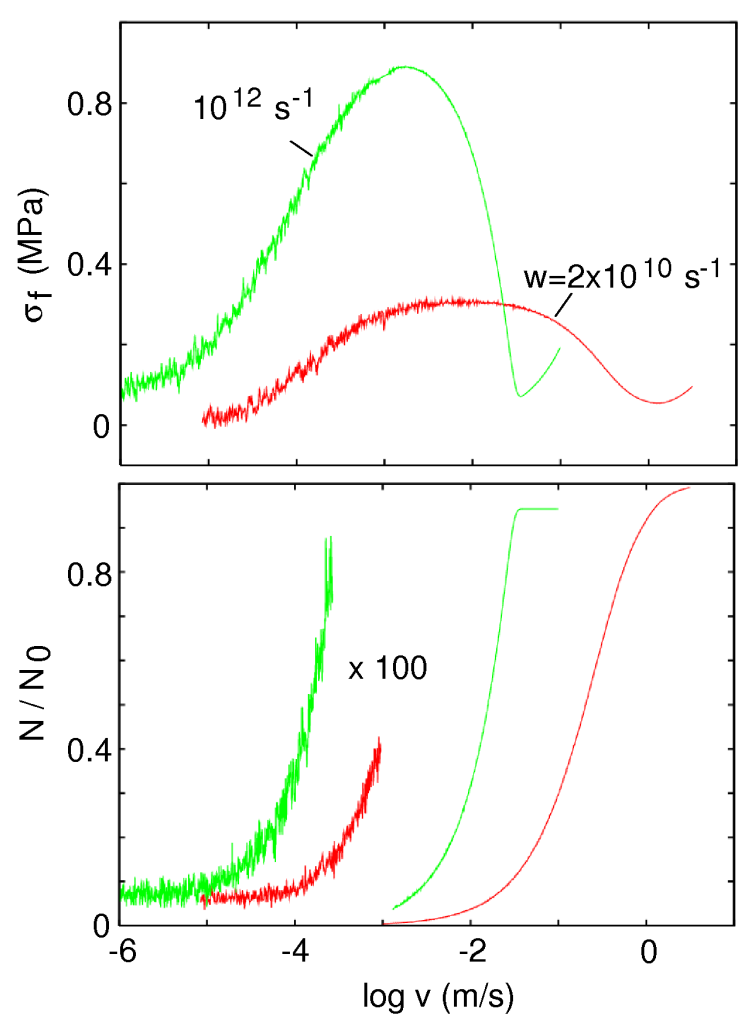

FIG. 9: The frictional shear stress $\sigma_{\mathrm{f}}$ as a function of the logarithm of the sliding velocity of the rubber block. For the temperature $T=20{ }^{\circ} \mathrm{C}$ and for the probability rates per unit time to return to the pinned state (when $\sigma<\sigma_{\mathrm{c} 1}$ ) $w=2 \times 10^{10} \mathrm{~s}^{-1}$ and $w=10^{12} \mathrm{~s}^{-1}$. All other parameters as in Fig. 5 .

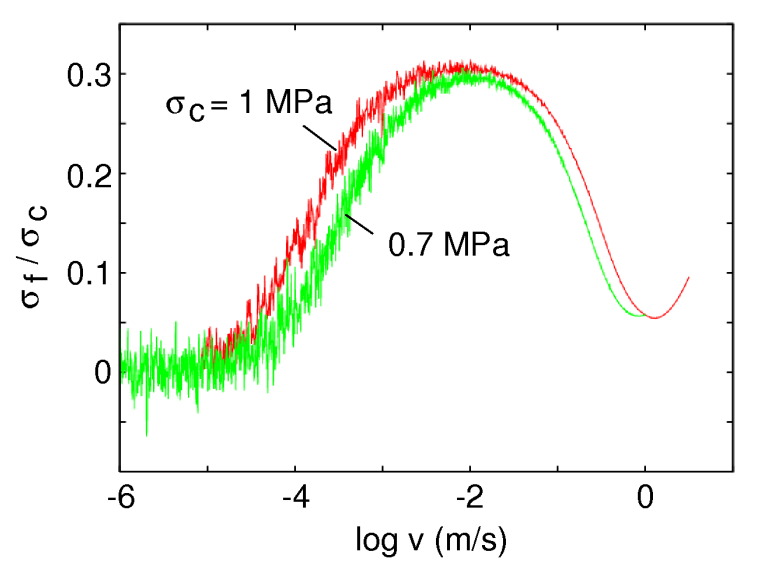

FIG. 10: The frictional shear stress $\sigma_{\mathrm{f}}$ as a function of the logarithm of the sliding velocity of the rubber block. For the temperature $T=20{ }^{\circ} \mathrm{C}$ and for the (zero-temperature) depinning stresses $\sigma_{\mathrm{c}}=1 \mathrm{MPa}$ and and $\sigma_{\mathrm{c}}=0.7 \mathrm{MPa}$. All other parameters as in Fig. 5. 


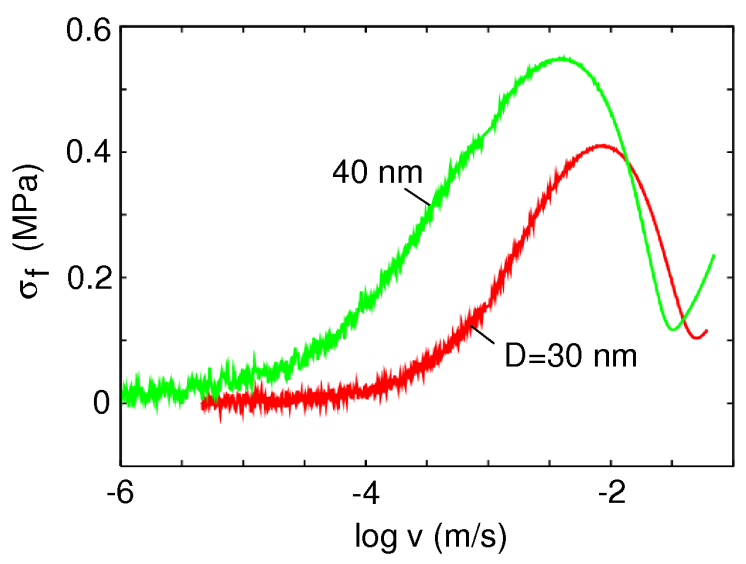

FIG. 11: The frictional shear stress $\sigma_{\mathrm{f}}$ as a function of the logarithm of the sliding velocity of the rubber block. For unfilled styrene butadiene (SB) copolymer for $T=20{ }^{\circ} \mathrm{C}$. In the calculation we have used the (zero-temperature) depinning stress $\sigma_{\mathrm{c}}=1 \mathrm{MPa}$ and the stress block size $D=30 \mathrm{~nm}$ and $40 \mathrm{~nm}$. The number of stress blocks was 128 . The viscous friction coefficient during steady sliding $\eta=0.03$ (natural units), and the critical stress below which steady sliding becomes metastable $\sigma_{\mathrm{c} 1}=0.1 \sigma_{\mathrm{c}}=0.1 \mathrm{MPa}$. The probability rate per unit time to return to the pinned state when $\sigma<\sigma_{\mathrm{c} 1}$ is $w=10^{12} \mathrm{~s}^{-1}$.

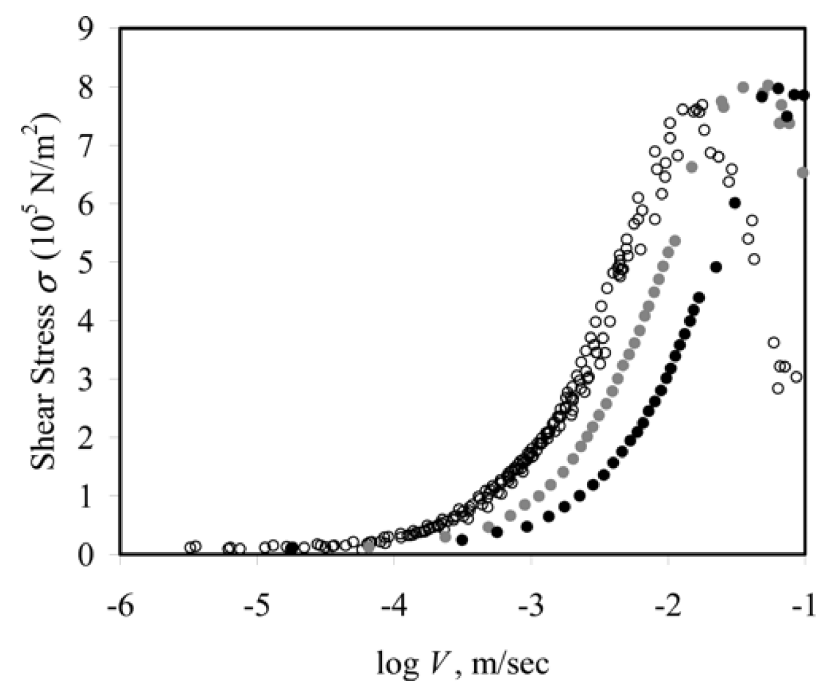

FIG. 12: Shear stress as a function of velocity and temperature for a silicon elastomer (low frequency elastic modulus $E \approx 5 \mathrm{MPa}$ ) sliding on a Si wafer covered by an inert selfassembled monolayers film. Open circles, gray circles and black circles represent data at 298, 318 and $348 \mathrm{~K}$, respectively. Adapted from Ref. [20]

for $v<v_{1}$, but not for $v>v_{1}$. Thus, the decrease in the friction which we observe for $v>v_{1}$ is nearly temperature independent. The difference between our prediction and the Grosch results can be understood as follows: Most likely, the decrease of the friction for $v>v_{1}$ in the Grosch experiment is related to a decrease in the area of real contact with increasing sliding velocity. The Grosch

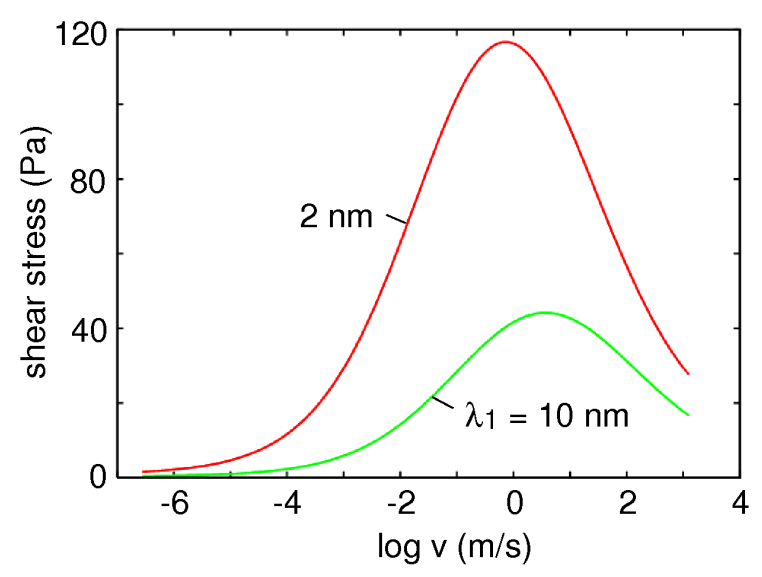

FIG. 13: The frictional shear stress $\sigma_{\mathrm{f}}$ as a function of the logarithm of the sliding velocity of the rubber block. For Styrene butadiene copolymer with $60 \%$ carbon black and for $T=40{ }^{\circ} \mathrm{C}$. The friction is entirely due to the surface roughness of the substrate which is assumed to be self affine fractal with the fractal dimension $D_{\mathrm{f}}=2.3$ and the root-meansquare roughness $0.5 \mathrm{~nm}$. The long-wavelength and short wavelength roll-off wavevectors $q_{0}=2 \pi / \lambda_{0}$ and $q_{1}=2 \pi / \lambda_{1}$ where $\lambda_{0}=100 \mu \mathrm{m}$ and $\lambda_{1}=2 \mathrm{~nm}$ (upper curve) and $10 \mathrm{~nm}$ (lower curve).

experiments where performed on smooth but wavy glass, and the area of real contact depends on the effective elastic modulus of the rubber. Thus, as the sliding velocity increases or, equivalently, the temperature decreases, the rubber becomes more stiff and the area of real contact decreases. Hence, if the shear stress remains constant at high sliding velocity [as we indeed observe in our calculations if the calculations are performed at low velocities and different temperatures, and shifted to higher velocity using the WLF equation], then the friction force will decrease with increasing sliding velocity because of the decrease in the area of real contact.

\section{The role of surface roughness: application to rubber sealing}

The experiments by Vorvolakos and Chaudhury 20] was performed on (passivated) silicon wafers with the root-mean-square roughness of at most $0.5 \mathrm{~nm}$. For such smooth surfaces the adhesive rubber-substrate interaction will, even in the absence of a squeezing pressure, give rise to complete contact between the rubber and the substrate within the nominal contact area. For this case we have applied the rubber friction theory developed in Ref. 2] to obtain the contribution to the frictional shear stress from the roughness induced bulk viscoelastic deformations in the rubber (Fig. 2.). We have assumed that the substrate is self affine fractal with the fractal dimension $D_{\mathrm{f}}=2.3$ and the long-distance roll off wavelength $\lambda_{0}=100 \mu \mathrm{m}$. We have included roughness components down to the short-distance (cut-off) wavelength $\lambda_{1}$. 


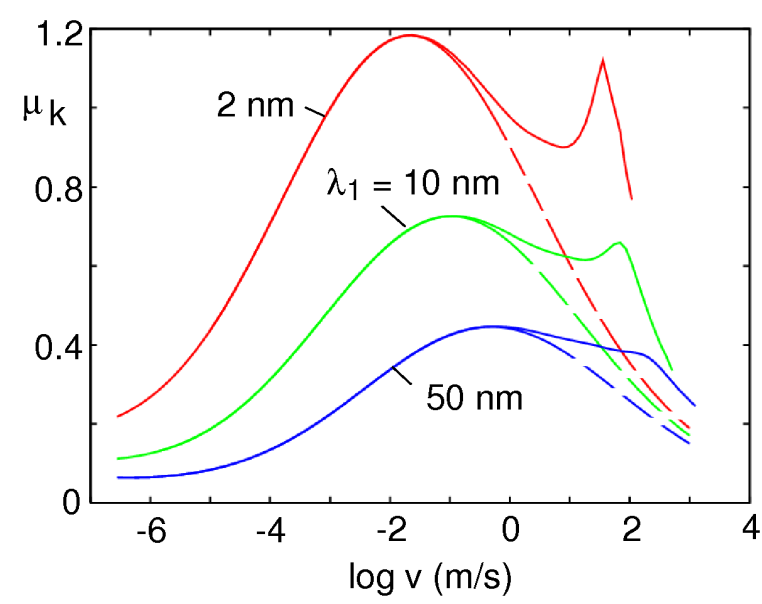

FIG. 14: The kinetic friction coefficient $\mu_{\mathrm{k}}$ as a function of the logarithm (with 10 as basis) of the sliding velocity of the rubber block. For Styrene butadiene copolymer with $60 \%$ carbon black and for $T=40{ }^{\circ} \mathrm{C}$. The friction is entirely due to the surface roughness of the substrate which is assumed to be self affine fractal with the fractal dimension $D_{\mathrm{f}}=2.3$ and the root-mean-square roughness $1 \mu \mathrm{m}$. The long-wavelength and short wavelength roll-off wavevectors $q_{0}=2 \pi / \lambda_{0}$ and $q_{1}=2 \pi / \lambda_{1}$ where $\lambda_{0}=100 \mu \mathrm{m}$ and $\lambda_{1}=2 \mathrm{~nm}$ (top curve), $10 \mathrm{~nm}$ (middle curve) and $50 \mathrm{~nm}$ (bottom curve). The solid curves are the full calculation while the dashed curves are without the flash temperature effect.

In Fig. 13 we show the resulting frictional shear stress (assuming perfect contact at the sliding interface) using $\lambda_{1}=10 \mathrm{~nm}$ and $2 \mathrm{~nm}$. The smallest possible $\lambda_{1}$ is determined by an atomic distance but a more likely cut-off length in the present case is the mean distance between rubber cross links, which is of order a few nanometer. The calculated maximal shear stress is of order $\sim 100 \mathrm{~Pa}$, which is a factor $\sim 10^{4}$ smaller than the shear stress observed in Ref. 20]. Hence we conclude that the surface roughness in the measurements of Vorvolakos and Chaudhury has a negligible influence on the observed frictional shear stress. However, the surfaces used in Ref. 20 are exceptionally smooth, and it is of great interest to study the influence of the surface roughness on rubber friction for surfaces of more common use in rubber applications.

One very important application of rubber is for seals. In a typical such application the rubber is sliding on a (lubricated) steel surface with the root-mean-square roughness of order $1 \mu \mathrm{m}$. At low sliding velocity (e.g., at the start of sliding) the lubricant fluid is squeezed away from the rubber-substrate asperity contact regions, giving friction coefficients similar to those of dry (unlubricated) surfaces, typically of order $\mu_{\mathrm{k}} \sim 0.5$.

Recently Mofidi et al 24 have performed rubber friction studies for nitrile rubber (the most popular seal material) in contact with a steel surface. The experiments was performed by squeezing a steel cylinder (diameter $1.5 \mathrm{~cm}$ and length $2.2 \mathrm{~cm}$ ) with the force $F_{\mathrm{N}}=100 \mathrm{~N}$

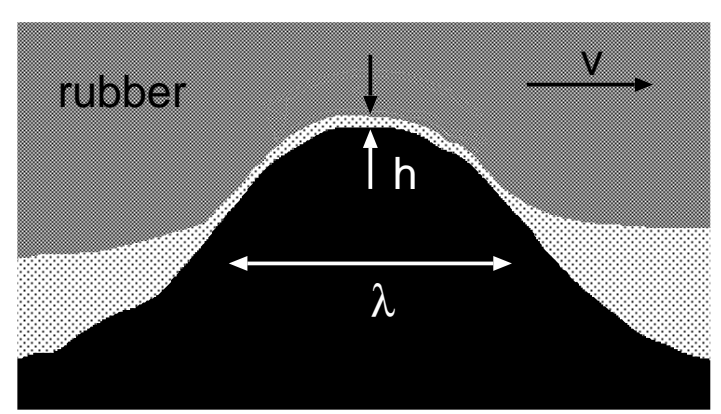

FIG. 15: Rubber in squeezed contact with a hard rough substrate. During sliding the bulk viscoelastic deformation (on the length scale $\lambda$ ) at an asperity contact region will not be influenced by the thin lubrication film (thickness $h$ ) at the asperity if $h<<\lambda$. The lubrication film will, however, remove the contribution to the friction from substrate asperities with height smaller than $h$.

against the nitrile rubber surface, lubricated by various types of oil with kinetic viscosities ranging from $\nu=5.5 \times 10^{-6}$ to $46 \times 10^{-6} \mathrm{~m}^{2} / \mathrm{s}$. The nominal squeezing pressure $p \approx 1 \mathrm{MPa}$. The steel cylinder was oscillating along its axis with the frequency $f=50 \mathrm{~Hz}$ and the amplitude $a=1 \mathrm{~mm}$. Thus, the average sliding velocity $\bar{v} \approx \omega a / \sqrt{ } 2 \approx 0.2 \mathrm{~m} / \mathrm{s}$ (where $\omega=2 \pi f$ is the angular frequency). The steel surface had the rms roughness $1.03 \mu \mathrm{m}$. The experiment showed that the kinetic friction $\mu_{\mathrm{k}} \approx 0.6$ was nearly independent of the lubrication oil viscosity. This indicate that the lubrication oils are nearly completely removed from the steel-rubber asperity contact regions and that the observed friction is due to the asperity induced deformation of the rubber surface 2]. For perfectly smooth surfaces, the thickness $h$ of the oil film in the contact region is easy to estimate using the equation [25, 26] $h \approx(\rho \nu A / p t)^{1 / 2}$, where $\rho$ is the oil mass density, $\nu$ the kinematic viscosity, $A$ the nominal contact area, and $t$ the squeezing time which in the study presented in Ref. 24 was 15 minutes. In the present case this gives $h \approx 20-60 \mathrm{~nm}$. The actual film thickness at the asperity contact regions (see Fig. 15) may be much smaller.

We now show that the rubber friction theory developed in Ref. 2], where the whole friction is attributed to the asperity-induced viscoelastic deformations of the rubber, can in fact explain the magnitude of the friction observed by Mofidi et al 24] (which is similar to the friction observed for rubber seals during start-up of sliding [27]). Since the sliding surfaces are embedded in oil we will assume that there is negligible adhesive interaction between the surfaces so that the area of real contact is determined mainly by the applied pressure. In Fig. [14 we show the calculated kinetic friction coefficient $\mu_{\mathrm{k}}$ as a function of the logarithm of the sliding velocity of the rubber block. In the calculation we have assumed styrene butadiene 
(SB) copolymer with $60 \%$ carbon black and $T=40^{\circ} \mathrm{C}$. The friction is entirely due to the surface roughness of the substrate which is assumed to be self affine fractal with the fractal dimension $D_{\mathrm{f}}=2.3$ and the root-mean-square roughness $1 \mu \mathrm{m}$. The long-wavelength roll-off wavevector $q_{0}=2 \pi / \lambda_{0}$ with $\lambda_{0}=100 \mu \mathrm{m}$, and the short wavelength cut-off wavevector $q_{1}=2 \pi / \lambda_{1}$ where $\lambda_{1}=2 \mathrm{~nm}$ (top curve), $10 \mathrm{~nm}$ (middle curve) and $50 \mathrm{~nm}$ (bottom curve). The solid curves are the full calculation while the dashed curves are without the flash temperature effect 22, 28]. For the $\lambda_{1}=50 \mathrm{~nm}$ case, according to the calculation the area of real contact between the rubber and the substrate is only of order $0.3 \%$ of the nominal contact area so the frictional shear stress (at the shortest scale $\sim 50 \mathrm{~nm}$ ) is of order $\mu_{\mathrm{k}} p / 0.003 \approx 100 \mathrm{MPa}$ which is close to the (ideal) rupture stress of rubber at short length scale (the rupture stress at the macroscopic scale is usually much smaller due to "large" crack-like defects in most rubber objects). Thus, one may expect rubber wear to occur when sliding under the present conditions, as was also observed in the study reported on in Ref. [24].

In the context of rubber sealing, the surfaces of the hard countermaterials (usually steel) are prepared to have surface roughness with a rms amplitude in the range $\sim 0.1-1 \mu \mathrm{m}$, which will trap lubrication oil at the interface. After a long time of stationary contact lubricant fluid will only occur in the substrate valleys, but at the onset of sliding lubrication fluid is dragged out from the valleys (or cavities) to form (at high enough sliding velocity) a thin lubrication film between the substrate asperities and the rubber surface; this will reduce the friction and the rubber wear.

The theories presented above predict that the rubber friction decreases for high enough sliding velocity. This may result in stick-slip motion (as often observed, e.g., for rubber wiper blades), and is a major problem in many practical applications as it may generate strong noise and wear, and may lead to device malfunction.

We note, as a curiosity, that the increase in the temperature in the asperity contact regions (flash temperature) increases the friction at high sliding velocity, see Fig. 14] This is opposite to some other rubber applications, e.g. for tires, where the flash temperature decreases the friction 2, 28] This difference is due to the fact that in the present case the temperature effect becomes important only at relative high sliding velocities, where the asperity-induced perturbing frequencies occur on the glassy-side of the viscoelastic loss peak (the $\tan \delta$ peak); as the temperature increases the rubber viscoelastic spectra shift towards lower frequencies, i.e., the perturbing frequencies will occur closer to the maximum of the $\tan \delta$-curve, and the friction will increase.

We note that many earlier studies of the adhesional contribution to rubber friction have used "polished" (steel) surfaces 21]. But such surfaces typically have surface roughness on many different length scales with a

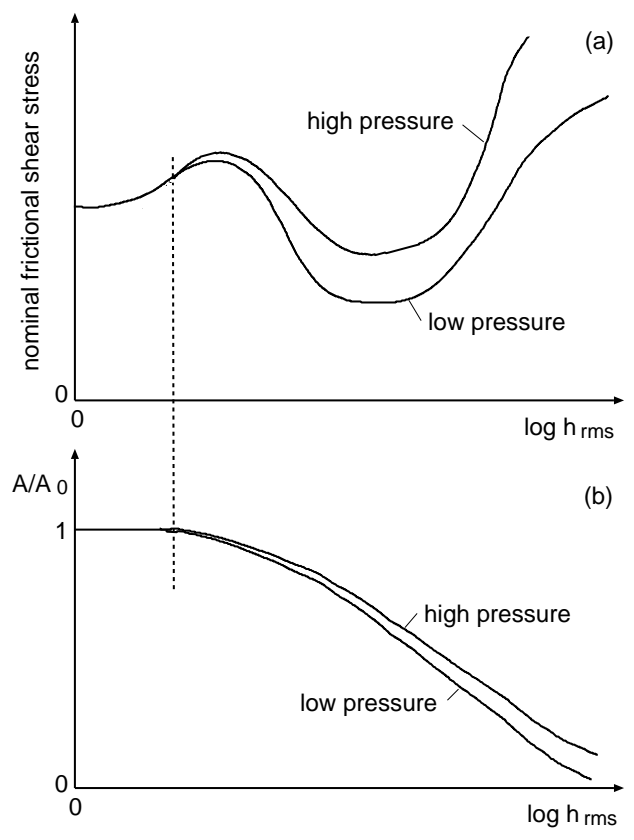

FIG. 16: The nominal frictional shear stress $\left(F_{\mathrm{f}} / A_{0}\right)(\mathrm{a})$ and the projected (on the $x y$-plane) rubber-substrate contact area $A$ (normalized by the nominal contact area $A_{0}$ ) (b) during sliding of a rubber block on substrates with increasing rootmean-square roughness $h_{\text {rms }}$. (Schematic.)

rms amplitude of order $1 \mu \mathrm{m}$ and cannot be considered as smooth with respect to rubber friction. In fact, as discussed above, the dominant contribution to the friction in these cases may be derived from the substrate asperity induced (viscoelastic) deformation of the rubber surface.

Finally, let us discuss (qualitatively) the magnitude of rubber friction as a function of increasing surface roughness. At small surface roughness, because of the rubbersubstrate adhesive interaction, there will be complete contact between the rubber and the substrate and the rubber friction will be the sum of the contributions illustrated in Fig. 2 (a) and (c), and the friction will increase with the surface roughness amplitude. As the roughness increases above some critical value, incomplete contact will occur at the sliding interface and when the surface roughness becomes large enough the friction may decrease. However at large enough roughness the rubber friction may increase again, at least if the (perpendicular) squeezing pressure is high enough. It is clear that even in the absence of a lubricant, for sealing-applications the best substrate surface may have some (small) roughness in order to minimize the friction.

\section{Summary and conclusion}

We have studied the sliding friction for viscoelastic solids, e.g., rubber, on hard flat substrate surfaces. We have shown that the fluctuating shear stress, which result from the thermal motion of the atoms or molecules 
in a viscoelastic solid, gives rise to very strong stressfluctuations, which at the nanoscale are in the MParange. This is similar to the depinning stresses which typically occur at solid-rubber interfaces, indicating the crucial importance of thermal fluctuations for rubber friction on smooth surfaces. We have developed a detailed model which takes into account the influence of thermal fluctuations on the depinning of small contact patches (stress domains) at the rubber-substrate interface. The theory predict that the velocity dependence of the macroscopic shear stress has a bell-shaped form, and that the low-velocity side exhibit the same temperature dependence as the bulk viscoelastic modulus, in qualitative agreement with experimental data. Finally, we have discuss the influence of small-amplitude substrate roughness on rubber sliding friction and shown that in typical applications to rubber sealing, the substrate asperity-induced viscoelastic deformations of the rubber surface may give the dominant contribution to the sliding friction.

\section{Acknowlengement:}

A.V. and B.P. thank DFG and Pirelli Pneumatici for support. A.V. and B.P. thank the EU for support within the "Natribo" network of the European Science Foundation.

\section{Appendix A: Memory friction and fluctuating} force

Eq. (11) is a standard result in the general theory of Brownian motion which can be derived in various ways. The most general proof is based on the memory function formalism as described, e.g., in the book by D. Forster 29]. A simpler (but less general) derivation of Eq. (11) involves the study of a particle (coordinate $q(t)$ ) coupled to an infinite set of harmonic oscillators (the heat bath) (coordinates $x_{\mu}$ ). For the readers convenience, we briefly review this derivation here. The particle and the heat bath coordinates satisfies the equations of motion

$$
\begin{gathered}
m \ddot{q}+\sum_{\mu} \alpha_{\mu} x_{\mu}=0 \\
m_{\mu} \ddot{x}_{\mu}+m_{\mu} \omega_{\mu}^{2} x_{\mu}+\alpha_{\mu} q+m_{\mu} \eta_{\mu} \dot{x}_{\mu}=f_{\mu}
\end{gathered}
$$

where

$$
\left\langle f_{\mu}(t) f_{\nu}\left(t^{\prime}\right)\right\rangle=2 m_{\mu} \eta_{\mu} k_{B} T \delta\left(t-t^{\prime}\right) \delta_{\mu \nu}
$$

If we define

$$
\begin{aligned}
x_{\mu}(t) & =\int d \omega x_{\mu}(\omega) e^{-i \omega t} \\
x_{\mu}(\omega) & =\frac{1}{2 \pi} \int d t x_{\mu}(t) e^{i \omega t}
\end{aligned}
$$

and similar for $q$ and $f_{\mu}$, we get from (A2)

$$
x_{\mu}(\omega)=\frac{f_{\mu}(\omega)-\alpha_{\mu} q(\omega)}{m_{\mu}\left(\omega_{\mu}^{2}-\omega^{2}-i \omega \eta_{\mu}\right)}
$$

and from (A3),

$$
\left\langle f_{\mu}(\omega) f_{\nu}\left(\omega^{\prime}\right)\right\rangle=m_{\mu} \eta_{\mu} k_{B} T \delta\left(\omega+\omega^{\prime}\right) \delta_{\mu \nu} / \pi .
$$

From (A4) we get

$$
\sum_{\mu} \alpha_{\mu} x_{\mu}(\omega)=\gamma(\omega) q(\omega)-f(\omega)
$$

where

$$
\gamma(\omega)=\sum_{\mu} \frac{\alpha_{\mu}^{2}}{m_{\mu}\left(\omega_{\mu}^{2}-\omega^{2}-i \omega \eta_{\mu}\right)}
$$

and

$$
f(\omega)=\sum_{\mu} \frac{\alpha_{\mu} f_{\mu}(\omega)}{m_{\mu}\left(\omega_{\mu}^{2}-\omega^{2}-i \omega \eta_{\mu}\right)}
$$

Using (A1) and $\mathrm{A}(6)$ gives

$$
-m \omega^{2} q(\omega)+\gamma(\omega) q(\omega)=f(\omega)
$$

Using (A8), (A7) and (A5) it is easy to show that

$$
\left\langle f(\omega) f\left(\omega^{\prime}\right)\right\rangle=-\frac{k_{B} T}{\pi \omega} \operatorname{Im} \gamma(\omega) \delta\left(\omega+\omega^{\prime}\right)
$$

Note also that (A9) is equivalent to

$$
m \ddot{q}+\int_{-\infty}^{t} d t^{\prime} \gamma\left(t-t^{\prime}\right) q\left(t^{\prime}\right)=f(t)
$$

In Sec. 3.1 we studied a system of many coupled dynamical variables

$$
m \ddot{q}_{i}+\int_{-\infty}^{t} d t^{\prime} \gamma_{i j}\left(t-t^{\prime}\right) q_{j}\left(t^{\prime}\right)=f_{i}(t)
$$

but this problem can be reduced to the problem studied above by forming new dynamical variables, as linear combination of the old dynamical variables $q_{i}$, chosen so that $\gamma_{i j}$ becomes diagonal.

[1] D. F. Moore, The Friction and Lubrication of Elastomers (Oxford: Pergamon, 1972)

[2] B.N.J. Persson, J. Chem. Phys. 115, 3840 (2001).

[3] B.N.J. Persson and A.I. Volokitin, Phys. Rev. B65, 134106 (2002).

[4] M. Klüppel, G. Heinrich, Rubber Chem. Technol. 73, 578 (2000).

[5] A. Schallamach, Wear 6, 375 (1963).

[6] Y.B. Chernyak and A.I. Leonov, Wear 108, 105 (1986). 
[7] A.E. Filippov, J. Klafter and M. Urbakh, Phys. Rev. Lett. 92, 135503 (2004).

[8] Weak absorption systems (e.g., saturated hydrocarbon molecules or noble gas atoms on metal surfaces) exhibit extremely low (pinning) barriers for parallel motion. The barriers can be estimated from the measured frequencies of parallel adsorbate vibrations, see. e.g., B.N.J. Persson, Surface Science Reports 15, 1 (1990).

[9] M.L. Williams, R.F. Landel and J.D. Ferry, J. Am. Chem. Soc. 77, 3701 (1955).

[10] B.N.J. Persson, Phys. Rev. B51, 13568 (1995).

[11] B.N.J. Persson, O. Albohr, G. Heinrich and H. Ueba, J. Phys. Condens. Matter 17, R1071 (2005).

[12] B.N.J. Persson and E. Brener, Phys. Rev. E71, 036123 (2005).

[13] B. N. J. Persson, Surf. Sci. 401, 445 (1998).

[14] A. Schallamach, Wear 17, 301 (1971).

[15] A.D. Roberts and A.G. Thomas, Wear 33, 45 (1975).

[16] U. Tartaglino, V.N. Samoilov and B.N.J. Persson, J. Phys. Condens. Matter 18, 4143 (2006).

[17] B.N.J. Persson, Surface Science Reports, in press.

[18] B.N.J. Persson, Phys. Rev. B63, 104101 (2001)

[19] The force acting on a stress domain should depend quasiperiodically on the lateral displacement $x$ i.e., $F=$ $F_{c} \sin (2 \pi x / a)$ giving for small $x: F \approx F_{\mathrm{c}} 2 \pi x / a$. The pinning stress $F_{\mathrm{c}}=\sigma_{\mathrm{c}} D^{2}$ and the elastic force $F=k x$ with $k=\alpha E D$ where $\alpha$ is of order $\sim 6$. Thus we get $E D \approx \sigma_{\mathrm{c}} D^{2} / a$.
[20] K. Vorvolakos and M.K. Chaudhury, Langmuir 19, 6778 (2003).

[21] K.A. Grosch, Proc. R. Soc. London, A274, 21 (1963); K. A. Grosch, in The Physics of Tire Traction: Theory and Experiment, D. F. Hays, A. L. Browne, Eds. (Plenum press, New York-London 1974), page 143.

[22] A. Casoli, M. Brendle, J. Schultz, A. Philippe and G. Reiter, Langmuir 17, 388 (2001).

[23] T. Baumberger and C. Caroli, Advances in Physics (in press); T. Baumberger, C. Caroli and O. Ronsin, Eur. Phys. JE11, 85 (2003); O. Ronsin and K.L. Coeyrehourcq, Proceedings of the Royal Society of London series A-Mathematical physical and engineering sciences 457, 1277 (2010).

[24] M.R. Mofidi, E. Kassfeldt and B. Prakash, to be published.

[25] B.N.J. Persson, Sliding Friction: Physical Principles and Application, 2nd ed.; Springer: Heidelberg, 2000.

[26] B.N.J. Persson and F. Mugele, J. Phys. J. Phys. Condens. Matter 16, R295 (2004).

[27] J. Molter, Elastomerreibung und Kontactmechanik, DIK fortbildungsseminar, Hannover 2003.

[28] B.N.J. Persson, subm. to J. Phys. Condens. Matter.

[29] D. Forster, Hydrodynamic Fluctuations, Broken Symmetry, and Correlation Functions Benjamin, London (1975). 